\title{
Nominal and Detailed LTE Radio Network Planning considering Future Deployment in Dhaka City
}

\author{
Nafiz Imtiaz Bin Hamid \\ Department of EEE \\ Islamic University of \\ Technology \\ Gazipur-1704, Bangladesh.
}

\author{
Md. Ashraful Hoque \\ Department of EEE \\ Islamic University of \\ Technology \\ Gazipur-1704, Bangladesh.
}

\author{
Kazi Khairul Islam \\ Department of EEE \\ Islamic University of \\ Technology \\ Gazipur-1704, Bangladesh.
}

\begin{abstract}
Long Term Evolution (LTE) is the next major step in mobile radio communications and is introduced in 3rd Generation Partnership Project (3GPP) Release 8. It is the last step toward the 4 th generation $(4 \mathrm{G})$ of radio technologies designed to increase the capacity and speed of mobile telephone networks. With industrial attachment very few radio planning works of LTE are going on. But because of certain commercial issues those works aren't widely available. Radio network planning is a very vital step for wireless communication technology. As standardization work of LTE is approaching the end line, it is high time to go for efficient radio network planning guideline for LTE. In LTE just like other cellular technologies, initial planning is normally guided by various industries and vendors at their own discretion. They aren't likely to disclose their advancements and findings. That makes the job even more challenging. As a result, going on with LTE radio network planning perspective is a well-chosen challenge and a certain hot topic in the current research arena. In this work, a detailed LTE radio network planning procedure has been elaborated which concentrates on nominal and detailed planning considering possible network implementation in the densely populated South-Asian city-Dhaka.
\end{abstract}

\section{General Terms}

Telecommunications, Wireless Networks.

\section{Keywords}

Radio Network Planning, Planning Tool, Coverage Prediction, Traffic Map, LTE Simulation

\section{INTRODUCTION}

Whenever new cellular technology is considered for mass deployment hundreds of its RF parameters go through tuning process with a view to find out optimum value. But this phase is time consuming and very costly. So, before commercial deployment if extensive simulation can be run this tuning phase can be facilitated in numerous ways. Cost can also be greatly minimized. That is the benefit of running simulation before mass commercial deployment. In this sub-continent LTE is expected to be commercially launched in Q4 of 2012. All these aim at proper radio network planning of LTE. So, looking for optimization of the vital parameters in the least possible time is a very challenging issue which will obviously help network operators in a greater extent.

the number of resources to be allocated to the PDCCH and how UEs should be efficiently signaled over the PDCCH is addressed in [8]. Resource allocation in LTE downlink and LTE PHY layer simulation aspects have been featured respectively in [8] and [9]. [10-16] are the 3GPP Technical Specifications related to this work. Link and system level
The main advantages with LTE are high throughput, low latency, plug and play, frequency division duplexing (FDD) and time division duplexing (TDD) in the same platform, an improved end-user experience and a simple architecture resulting in low operating costs. LTE downlink transmission scheme is based on Orthogonal Frequency Division Multiple Access (OFDMA) - which converts the wide-band frequency selective channel into a set of many at fading subchannels. The LTE specification provides downlink peak rates of at least $100 \mathrm{Mbps}$ and an uplink of at least $50 \mathrm{Mbps}$. LTE supports scalable carrier bandwidths, from $1.4 \mathrm{MHz}$ to 20 $\mathrm{MHz}$ and supports both FDD and TDD [1] [2] [3]. LTE will also support seamless passing to cell towers with older network technology such as GSM, CDMA-One, W-CDMA (UMTS), and CDMA2000 [1, 2] [4, 5].

Radio network planning being quite a vital step for a wireless communication technology and as its standardization work is approaching the end line; it is high time to go for efficient radio network planning guideline. For the same reason, along with the fact that in LTE radio network planning just like other cellular technologies, initial stage planning is normally guided by various industries and vendors at their own discretion; they aren't likely to disclose their advancements and findings. That makes the job even more challenging.

The ultimate objective of this work is to come up with the detailed radio network planning guideline with respect to Dhaka city. With this mission ahead, in this paper a step by step method has been followed using radio planning tool Atoll. The idea was to cover the nominal and detailed planning stage in detail with respect to Dhaka city. Performance analysis of the planned network has also been included here. Prior to that, a brief description of the nominal and detailed radio planning has been given.

\section{RELATED WORKS}

In [6] coverage and capacity estimation is carried out in radio network dimensioning. Radio link budget is investigated for coverage planning. Theoretical work is later put into the development of an Excel based dimensioning tool which is designed to keep the interface simple and to set the functional parts clearly distinguishable. The final product gives the number of sites (cells) needed in order to support a certain subscriber population with a given capacity. In [7] an attempt to provide analysis of LTE system performance from radio network planning aspects has been made. Determination of simulation results have been obtained using [17] and [18] respectively. Effect of change in number of transmitting antennas has been shown in [19]. An attempt to facilitate a planned decision making stage for the mobile broadband solution specifically focusing in the South Asian region has been done in [20]. In [21] a detailed LTE radio network 
dimensioning procedure i.e. capacity and coverage analysislink budget preparation, link and system level simulation; has been performed in order to prepare a radio planning guideline considering possible network implementation in Dhaka city.

\section{RADIO NETWORK PLANNING PROCESS}

Radio Network Planning contains number of phases:

- Initial phase-which includes collection of pre-planning information and starting network dimensioning i.e. Link Budget preparation, coverage and capacity calculation by running simulations.

- Nominal and detailed planning- which includes selection and use of radio planning tool. This step involves propagation model tuning, defining thresholds from Link budget, creating detailed radio plan based on the thresholds, checking network capacity against more detailed traffic estimates, Configuration planning, Site surveys, Site pre-validation and validation, eNodeB parameter planning.

- Defining KPIs and Parameter Planning- using eNodeB system parameters and counters, defining performance KPIs and its target values based on vendor's promise, verification of the KPIs and target values using planning and dimensioning tools nominally along with pre and post-launch optimization.

But defining KPI and parameter planning has been considered out of the scope of this paper.

\section{RADIO PLANNING FOR DHAKA CITY}

Dhaka is the capital of Bangladesh and it is an overpopulated city in the South-Asian region. Efficient radio network planning is obviously a big challenge here with the optimal utilization of limited resources. In [21] coverage analysis i.e. link level simulation result along with link budget preparation and capacity analysis-system level simulation have been performed. Taking related pre-planning information of Dhaka: population: 15 million ( $0.75 \%$ to be covered), assumed overbooking factor: 50 , area: $1463.6 \mathrm{~km}^{2}$ in terms of CostHata propagation model no. of eNodeB for coverage was found as 53 while for capacity it was found as 50 . In this case, number of cell required for coverage exceeds that of capacity which means capacity can be effectively handled. The target capacity and coverage values are here attempted in the nominal and detailed radio planning stage involving radio planning tool-Atoll.

\section{ATOLL SIMULATIONS}

Digital map of Dhaka (shown in Fig.1) has been used for radio planning in this stage. These maps consisted of Dhaka airport, main road, secondary road, street, railway and water. At first to cover the whole Dhaka city eNodeBs were placed (shown in Fig 3) where the no. comes from the coverage and capacity analysis performed in [21]. After placing the eNodeBs coverage prediction was done that helped to justify the placement of the eNodeBs. Traffic maps were created for each of the Dhaka map subsections. Automatic frequency

planning and automatic cell planning were performed before running each of these simulations. In detail simulation result is obtained which contains: connected UL+DL, connected DL, connected UL, No service, Scheduler saturation, Resource saturation cases. Legends show each of them with different color. A separate table shows the simulation properties for each of the simulated traffic maps.

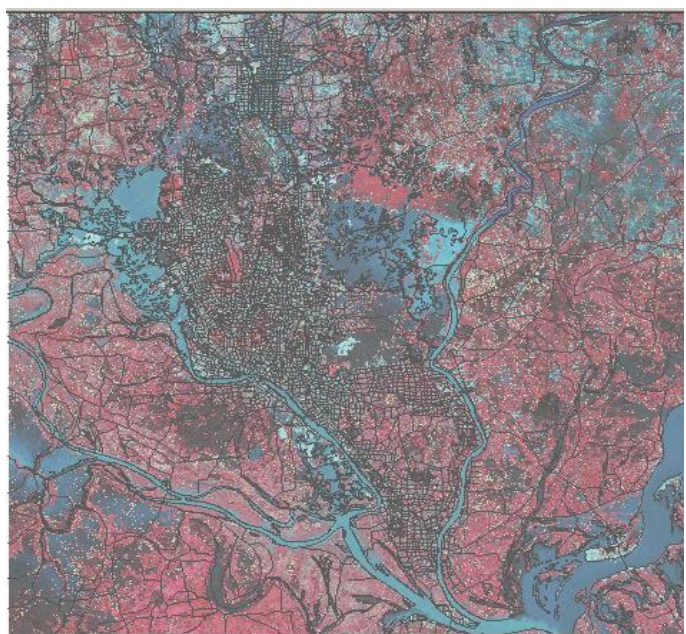

Fig 1: Dhaka Digital Map

\subsection{Coverage Prediction}

Coverage predictions have been performed by: transmitter, signal level, downlink throughput and Channel to Interference plus Noise Ratio (CINR). Coverage prediction properties: (a) by signal level, (b) channel throughput (DL) and (c) Downlink $\mathrm{C} /(\mathrm{I}+\mathrm{N})$ have been shown in Fig. 2. Corresponding coverage prediction results have been shown in Fig 4 to Fig 7.

(a)

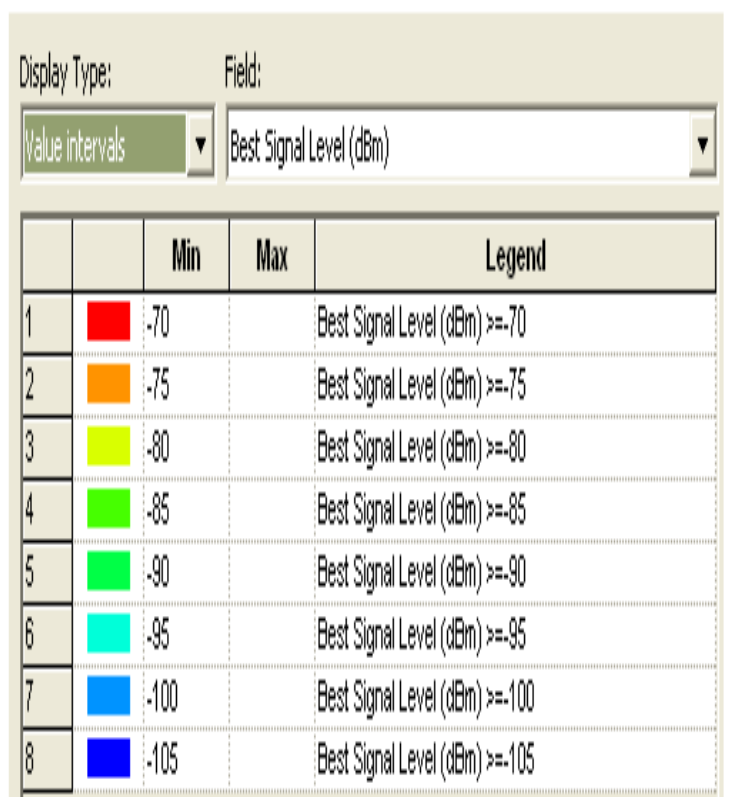




\begin{tabular}{|c|c|c|c|}
\hline \multicolumn{2}{|c|}{ Display Type: } & \multicolumn{2}{|l|}{ Field: } \\
\hline \multicolumn{2}{|c|}{ Walue intervals } & \multicolumn{2}{|c|}{ Peak RLC Channel Throughput (DL) (kbps) } \\
\hline & Min & Max & Legend \\
\hline 1 & 20,000 & & Peak RLC Channel Throughput (DL) (kbps) $:=20,000$ \\
\hline 2 & 19,000 & & Peak RLC Channel Throughpud (DL) (kbps) $>=19,000$ \\
\hline 3 & 18,000 & & Peak RLC Channel Throughpu (DL) (kbps) $s=18,000$ \\
\hline 4 & 17,000 & & Peak RLC Channel Throughput (DL) (kbps) $:=17,000$ \\
\hline 5 & 16,000 & & Peak RLC Channel Throughput (DL) (kbps) $>=16,000$ \\
\hline 6 & 15,000 & & Peak RLC Channel Throughput (DL) (kbps) $:=15,000$ \\
\hline 7 & 14,000 & & Peak RLC Channel Throughput (DL) (kbps) $>=14,000$ \\
\hline 8 & 13,000 & & Peak RLC Channel Throughput (DL) (kbps) $:=13,000$ \\
\hline 9 & 12,000 & & Peak RLC Channel Throughput (DL) (kbps) $>=12,000$ \\
\hline 10 & 11,000 & & Peak RLC Channel Throughput (DL) (kbps) $>=11,000$ \\
\hline 11 & 10,000 & & Peak RLC Channel Throughput (DL) (kbps) $>=10,000$ \\
\hline 12 & 9,000 & & Peak RLC Channel Throughput (DL) (kbps) s=9,000 \\
\hline 13 & 8,000 & & Peak RLC Channel Throughput (DL) (kbps) s=8,000 \\
\hline 14 & 7,000 & & Peak RLC Channel Throughput (DL) (kbps) s=7,000 \\
\hline$\overline{15}$ & 6,000 & & Peak RLC Channel Throughput (DL) (kbps) s=6,000 \\
\hline 16 & 5,000 & & Peak RLC Channel Throughput (DL) (kbps) $>=5,000$ \\
\hline 17 & 4,000 & & Peak RLC Channel Throughput (DL) (kbps) $>=4,000$ \\
\hline 18 & 3,000 & & Peak RLC Channel Throughput (DL) (kbps) s=3,000 \\
\hline 19 & 2,000 & & Peak RLC Channel Throughput (DL) (kbps) s=2,000 \\
\hline 20 & 1,000 & & Peak RLC Channel Throughput (DL) (kbps) s=1,000 \\
\hline
\end{tabular}

(b)

\begin{tabular}{|c|c|c|c|c|}
\hline \multicolumn{3}{|c|}{ Display Type: } & \multirow{2}{*}{\multicolumn{2}{|c|}{$\begin{array}{l}\text { Field: } \\
\text { PDSCH \& PDCCH C/(I+N) Level (DL) (dB) }\end{array}$}} \\
\hline \multicolumn{2}{|c|}{ Value intervals } & - & & \\
\hline & & Min & Max & Legend \\
\hline 1 & ㅍ & 30 & & PDSCH \& PDCCH Cr(I+N) Level $(\mathrm{DL})(\mathrm{dB})>=30$ \\
\hline 2 & 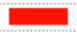 & 29 & & PDSCH \& PDCCH Cr(I+N) Level (DL) $(\mathrm{dB})==29$ \\
\hline 3 & $\mathbf{z}$ & 28 & & PDSCH \& PDCCH Cr(l+N) Level (DL) $(\mathrm{dB})>=28$ \\
\hline 4 & & 27 & & PDSCH \& PDCCH Cr(I+N) Level (DL) $(\mathrm{dB})>=27$ \\
\hline 5 & $\square$ & 26 & & PDSCH \& PDCCH Cr(I+N) Level (DL) $(\mathrm{dB})>=26$ \\
\hline 6 & $=$ & 25 & & PDSCH \& PDCCH Cr(I+N) Level (DL) (dB) $>=25$ \\
\hline 7 & 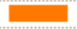 & 24 & & PDSCH \& PDCCH Cr(I+N) Level (DL) $(\mathrm{dB})>=24$ \\
\hline 8 & $=$ & 23 & & PDSCH \& PDCCH CI(I+N) Level (DL) (dB) $>=23$ \\
\hline 9 & Dans & 22 & & PDSCH \& PDCCH C(I+N) Level (DL) (dB) $>=22$ \\
\hline 10 & 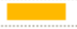 & 21 & & PDSCH \& PDCCH C (I+N) Level (DL) (dB) $>=21$ \\
\hline 11 & 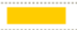 & 20 & & PDSCH \& PDCCH C(I+N) Level $(\mathrm{DL})(\mathrm{dB})==20$ \\
\hline 12 & 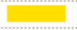 & 19 & & PDSCH \& PDCCH C(I+N) Level (DL) (dB) $>=19$ \\
\hline 13 & 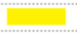 & 18 & & PDSCH \& PDCCH C $(1+N)$ Level $(D L)(d B)>=18$ \\
\hline 14 & $=$ & 17 & & PDSCH \& PDCCH C(I+N) Level $(\mathrm{DL})(\mathrm{dB})>=17$ \\
\hline 15 & $+a$ & 16 & & PDSCH \& PDCCH C $(1+N)$ Level $(D L)(d \mathrm{~d})>=16$ \\
\hline $\begin{array}{l}16 \\
17 \\
\end{array}$ & 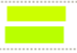 & 15 & & $\begin{array}{l}\text { PDSCH \& PDCCH CII+N) Level }(D L)(\mathrm{dB})>=15 \\
\text { PDSCH \& PDCCH C(I+N) Level (DL) }(\mathrm{dB})>=14\end{array}$ \\
\hline 18 & 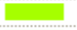 & 13 & & PDSCH \& PDCCH C(I+N) Level (DL) $(\mathrm{dB})>=13$ \\
\hline 19 & 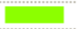 & 12 & & PDSCH \& PDCCH C(I+N) Level $(\mathrm{DL})(\mathrm{dB})>=12$ \\
\hline 20 & 5 & 11 & & PDSCH \& PDCCH C (1+N) Level (DL) (dB) $>=11$ \\
\hline 21 & 5 & 10 & & PDSCH \& PDCCH C(I+N) Level (DL) $(\mathrm{dB})==10$ \\
\hline 22 & 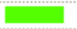 & 9 & & PDSCH \& PDCCH C(I+N) Level $(\mathrm{DL})(\mathrm{dB})>=9$ \\
\hline 23 & 5 & 8 & & PDSCH \& PDCCH CI(I+N) Level (DL) (dB) $>=8$ \\
\hline 24 & -1 & 7 & & PDSCH \& PDCCH Cr(I+N) Level (DL) (dB) $>=7$ \\
\hline 25 & 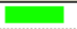 & 6 & & PDSCH \& PDCCH Cr(I+N) Level (DL) $(\mathrm{dB})>=6$ \\
\hline 26 & 5 & 5 & & PDSCH \& PDCCH CI(I+N) Level (DL) (dB) $>=5$ \\
\hline 27 & wann & 4 & & PDSCH \& PDCCH CI (I+N) Level (DL) (dB) $>=4$ \\
\hline 28 & $=$ & 3 & & PDSCH \& PDCCH Cr(I+N) Level (DL) (dB) $>=3$ \\
\hline 29 & = & 2 & & PDSCH \& PDCCH Cr(I+N) Level (DL) (dB) $>=2$ \\
\hline 30 & -1 & 1 & & PDSCH \& PDCCH Cr(I+N) Level $(D L)(d B)==1$ \\
\hline 31 & 5 & 0 & & PDSCH \& PDCCH Cr(I+N) Level (DL) (dB) $>=0$ \\
\hline 32 & $=$ & -1 & & PDSCH \& PDCCH C(I+N) Level (DL) (dB) $>=-1$ \\
\hline 33 & = & -2 & & PDSCH \& PDCCH Cl(I+N) Level (DL) (dB) $>=-2$ \\
\hline 34 & 5 & -3 & & PDSCH \& PDCCH C (1+N) Level (DL) (dB) $>=-3$ \\
\hline 35 & 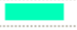 & -4 & & PDSCH \& PDCCH C(I+N) Level (DL) $(\mathrm{dB})>=-4$ \\
\hline 36 & 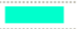 & -5 & & PDSCH \& PDCCH C(I+N) Level $(\mathrm{DL})(\mathrm{dB})>=-5$ \\
\hline
\end{tabular}

(c)

Fig 2: Coverage Prediction Properties by: (a) Signal Level (b) Channel Throughput $\quad$ (c) C/ (I+N) Level (DL)

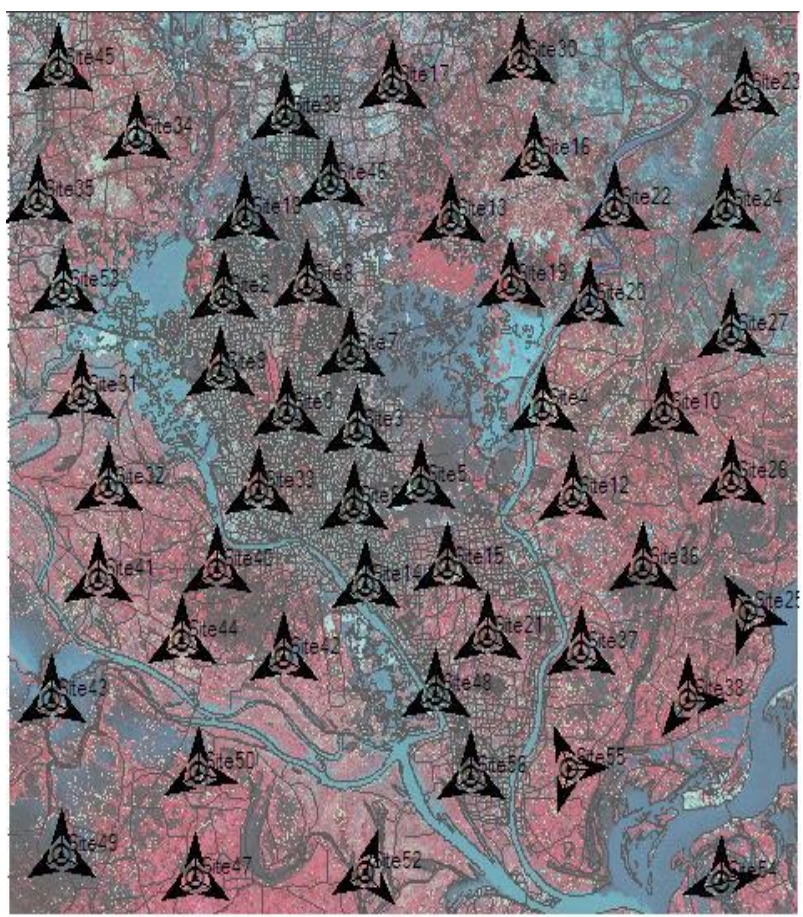

Fig 3: Transmitter Placed on Dhaka Map

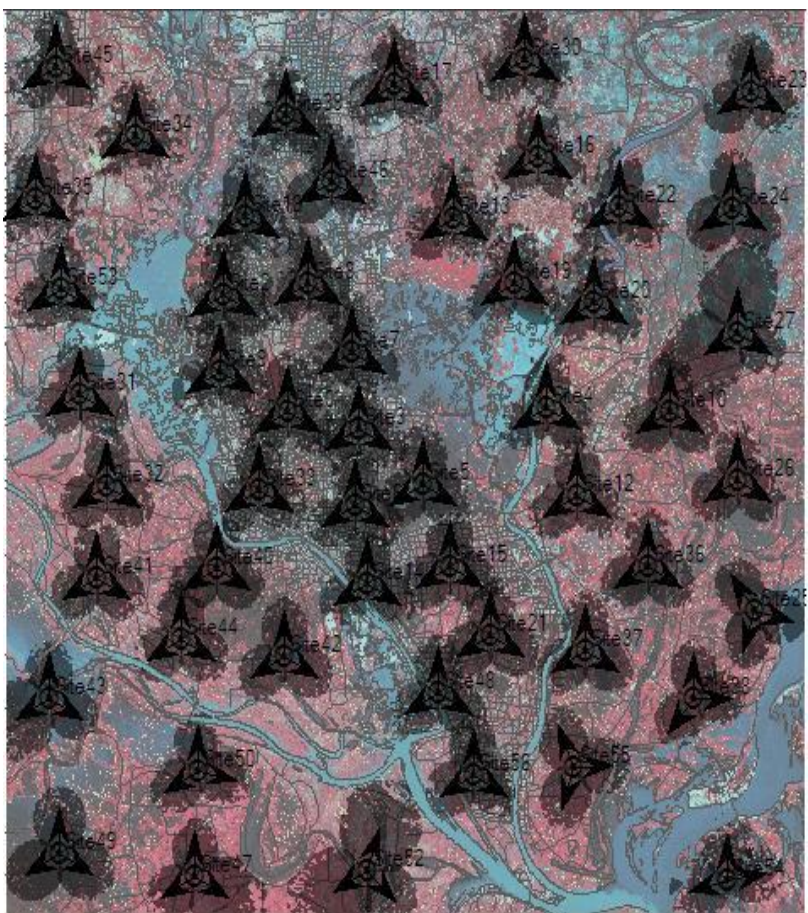

Fig 4: Coverage Prediction by Transmitter 


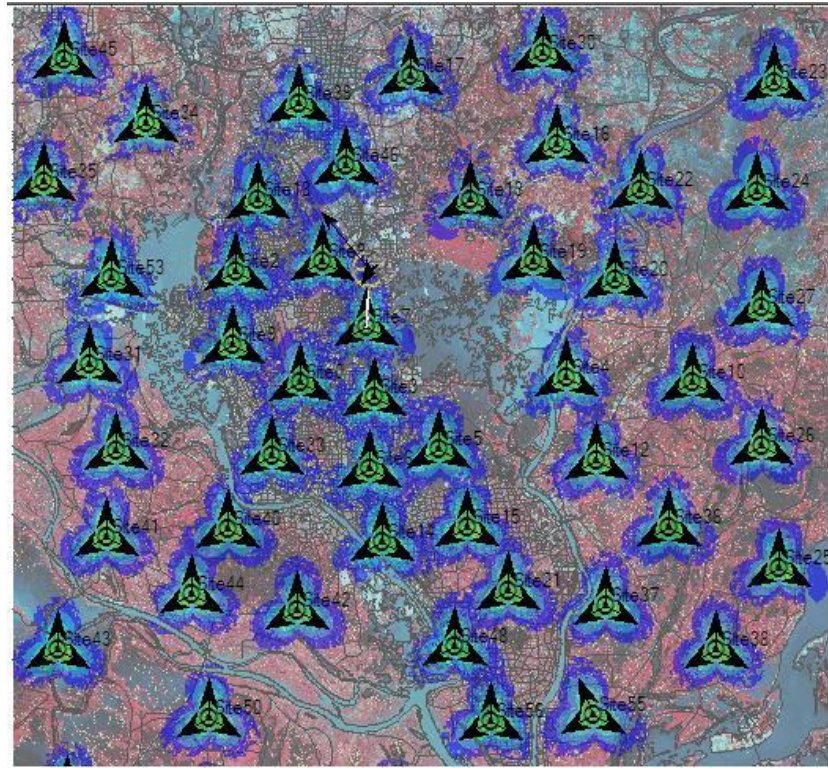

Fig 5: Coverage Prediction by Signal Level

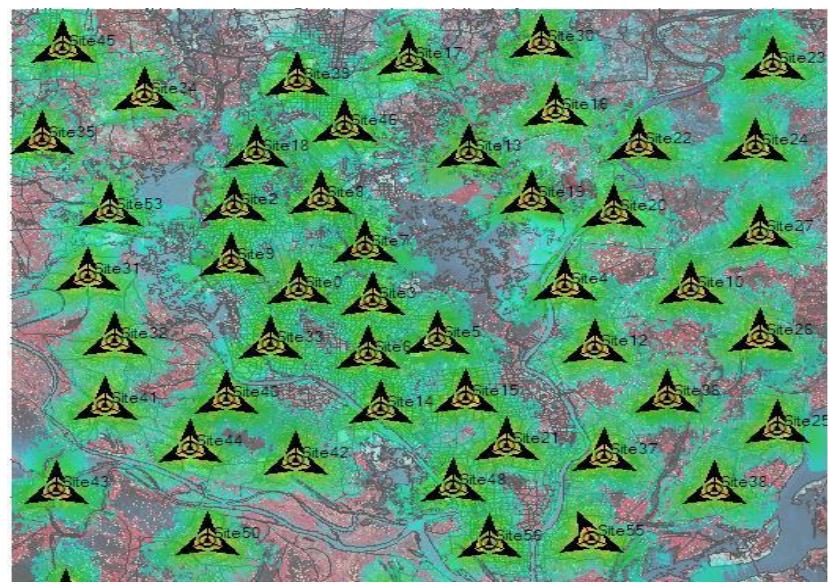

Fig 7: Coverage Prediction by CINR (DL)

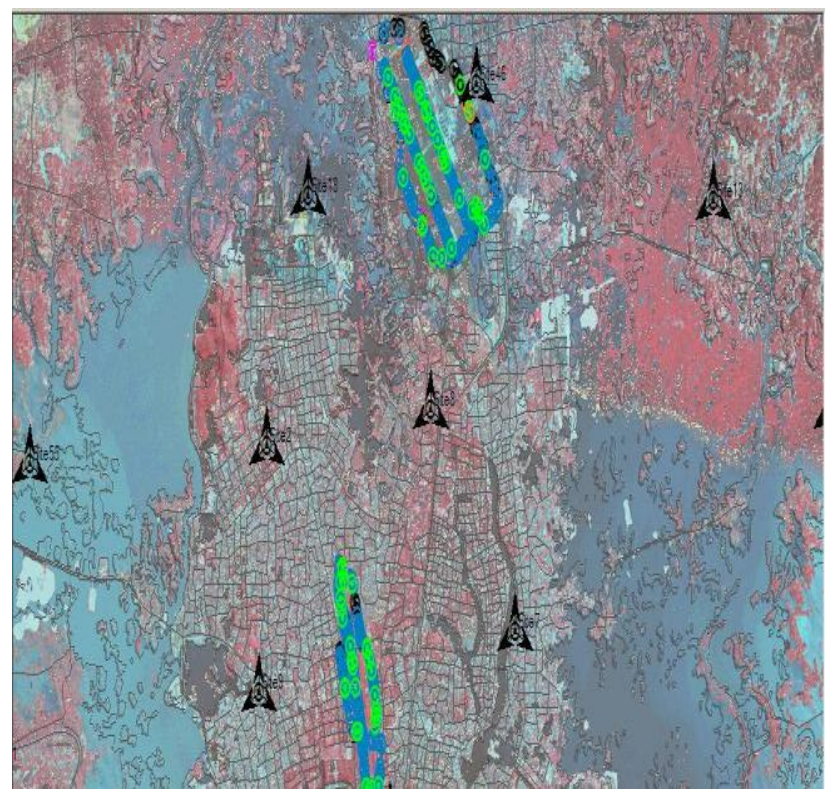

Fig 9(a): Dhaka Airport Traffic Map after Simulation

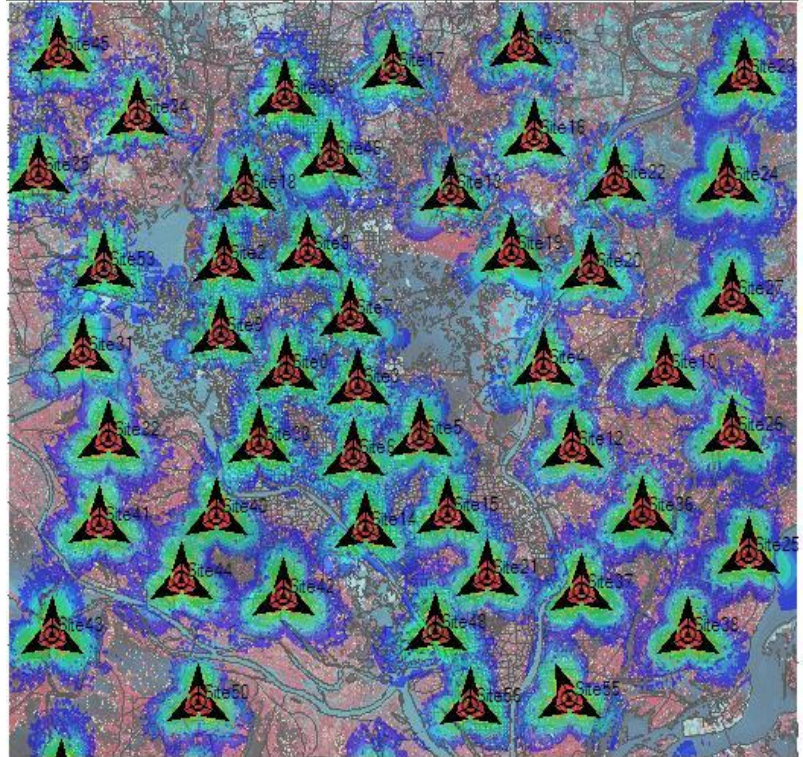

Fig 6: Coverage Prediction by Throughput (DL)

\begin{tabular}{|c|c|c|c|c|}
\hline \multicolumn{5}{|c|}{ Display | } \\
\hline \multicolumn{3}{|c|}{ Display Type: } & \multicolumn{2}{|c|}{ Field: } \\
\hline \multicolumn{2}{|c|}{ Discrete values } & - Conne & & 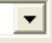 \\
\hline & & Value & Legend & \\
\hline 1 & $\odot$ & Connected DL+UL & Connected DL+UL & \\
\hline 2 & $\odot$ & Connected DL & Connected DL & \\
\hline 3 & $\odot$ & Connected UL & Connected UL & \\
\hline 4 & $\odot$ & No Service & No Service & \\
\hline 5 & $\odot$ & Scheduler Saturation & Scheduler Saturation & \\
\hline 6 & $\odot$ & Resource Saturation & Resource Saturation & \\
\hline
\end{tabular}

Fig 8: LTE Simulation Properties

\begin{tabular}{|c|c|c|c|c|c|}
\hline Service & $\begin{array}{l}\text { Reference } \\
\text { Cell }\end{array}$ & $\begin{array}{l}\text { Total } \\
\text { Path } \\
\text { Loss } \\
\text { (DL) } \\
\text { (dB) }\end{array}$ & $\begin{array}{l}\text { Application } \\
\text { Channel } \\
\text { Throughput } \\
\text { (DL) } \\
\text { (kbps) }\end{array}$ & $\begin{array}{c}\text { Transmission } \\
\text { Power } \\
\text { (UL) } \\
\text { (dBm) }\end{array}$ & $\begin{array}{l}\text { Reference } \\
\text { Signal } \\
\mathrm{C} /(\mathrm{I}+\mathrm{NI}) \\
\text { (DL) } \\
\text { (dB) }\end{array}$ \\
\hline FTP Download & Site $46 \_1(0)$ & 151.49 & 0 & 23 & -19.67 \\
\hline FTP Download & Site $46 \_1(0)$ & 153.59 & 0 & 23 & -8.74 \\
\hline FTP Download & Site $46 \_1(0)$ & 165.39 & 0 & 23 & -9.36 \\
\hline FTP Download & Site18_1 $1(0)$ & 163.13 & $2,706.7$ & 22.5 & -0.96 \\
\hline FTP Download & Site18_1 (0) & 171.38 & 0 & 23 & -10.06 \\
\hline VolP & Site $39{ }_{3} 3(0)$ & 156.43 & 0 & 23 . & -23.5 \\
\hline Web Browsing & Site $46 \_3(0)$ & 156.72 & 990.34 & 22.5 . & -5 \\
\hline FTP Download & Site18_2(0) & 176.48 & 0 & 23 & -19.84 \\
\hline FTP Download & Site $46 \_3(0)$ & 154.1 & $3,911.94$ & 22.9 & 0.16 \\
\hline FTP Download & & & 0 & 0 & \\
\hline Web Browsing & Site $46 \_3(0)$ & 155.64 & $3,911.94$ & 22.9 & 0.12 \\
\hline VolP & Site $39 \_2(0)$ & 175.9 & 0 & 23. & -26.68 \\
\hline FTP Download & Site18_2(0) & 176.92 & 0 & 23 & -13.35 \\
\hline FTP Download & Site $46 \_3(0)$ & 159.02 & 0 & 23 & -9.79 \\
\hline FTP Download & Site18_2 (0) & 166.28 & 0 & 23. & -15.75 \\
\hline Web Browsing & Site $46 \_3(0)$ & 166.32 & 0 & 23 & -13.87 \\
\hline FTP Download & Site $46 \_3(0)$ & 174 & 0 & 23 & -15.33 \\
\hline Web Browsing & Site18_1 (0) & 152.05 & 947.28 & 22.2 & -4.51 \\
\hline FTP Download & Site $46 \_3(0)$ & 167.31 & 0 & 23 & -28.4 \\
\hline FTP Download & Site $46 \_3(0)$ & 154.65 & 0 & 23 & -7.9 \\
\hline VoIP & Site $46 \_3(0)$ & 163.31 & $3,911.94$ & 22.5 & 0.54 \\
\hline VolP & Site $46 \_3(0)$ & 163.54 & 0 & 23 & -9.65 \\
\hline FTP Download & Site $46 \_3(0)$ & 157.13 & 0 & 23 & -10.95 \\
\hline Video Conferencing & Site $46 \_3(0)$ & 155.43 & $9,601.68$ & 23 & 7.55 \\
\hline FTP Download & Site $46 \_3(0)$ & 157.32 & 0 & 23 . & -10.78 \\
\hline
\end{tabular}

Fig 9(b): Dhaka Airport Traffic Map Simulation Properties 


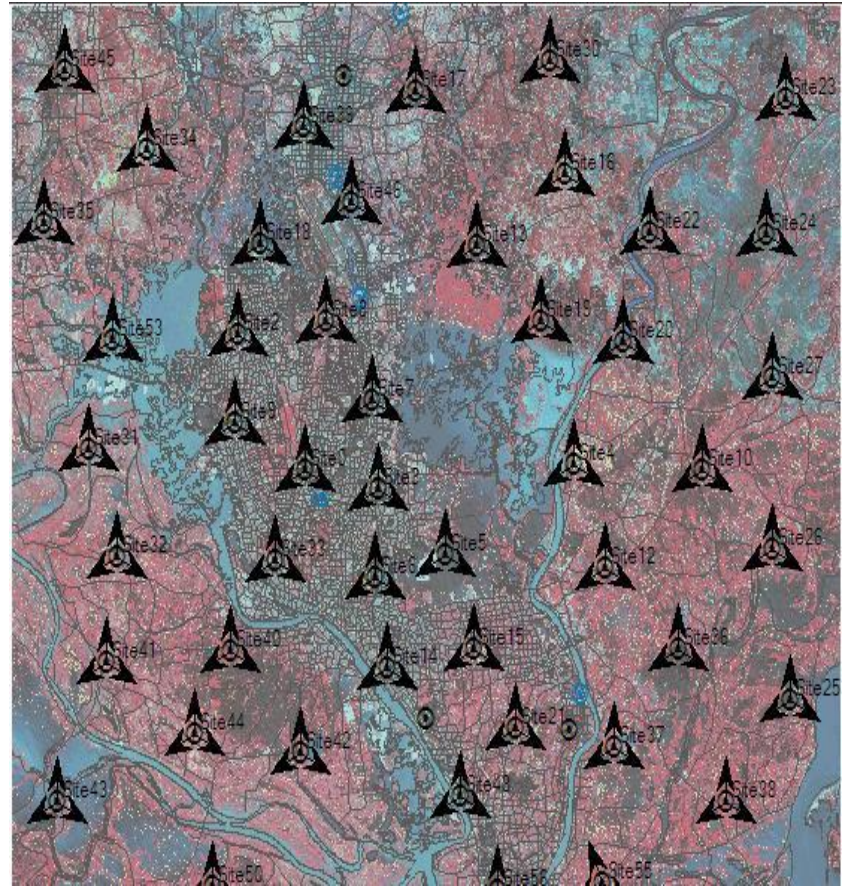

Fig 10(a): Dhaka Railway Traffic Map after Simulation

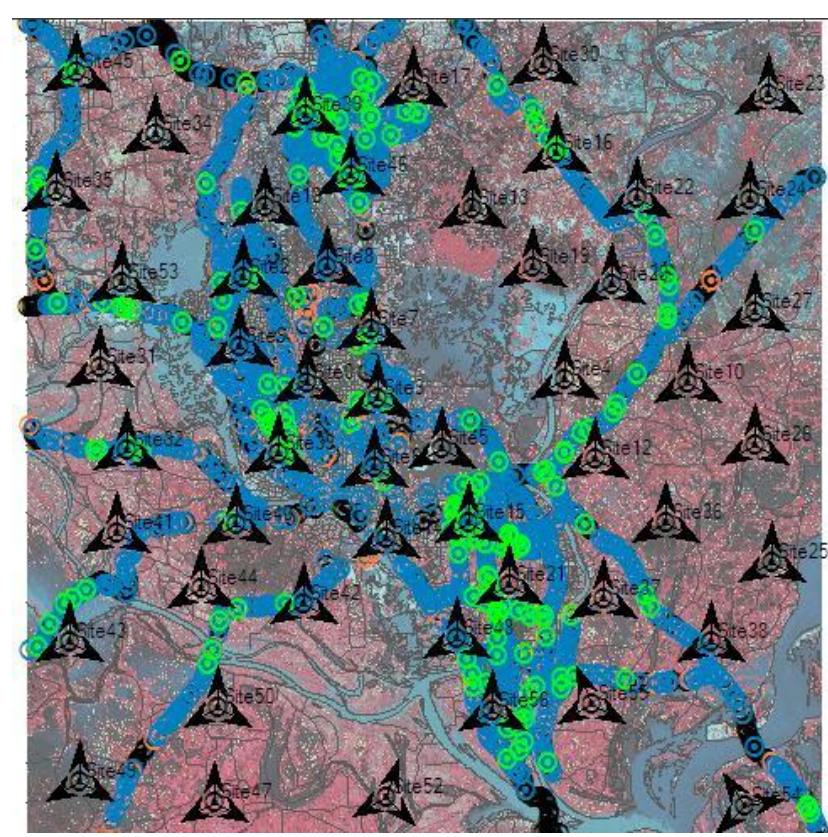

Fig 11(a): Dhaka Mainroad Traffic Map after Simulation

\begin{tabular}{|c|c|c|c|c|c|c|}
\hline Service & $\begin{array}{l}\text { Serving } \\
\text { Brse } \\
\text { Station }\end{array}$ & $\begin{array}{l}\text { Total } \\
\text { Path } \\
\text { Loss } \\
\text { (DL) } \\
\text { (dB) }\end{array}$ & $\begin{array}{l}\text { BLER } \\
\text { (DL) }\end{array}$ & $\begin{array}{c}\text { Trangmisgion } \\
\text { Powe } \\
\text { (UL) } \\
\text { (dBm) }\end{array}$ & $\begin{array}{c}\text { Application } \\
\text { User } \\
\text { Throughout } \\
\text { (DL) } \\
\text { (kops) }\end{array}$ & $\begin{array}{c}\text { Received } \\
\text { Reflerence } \\
\text { Signal } \\
\text { Power } \\
\text { (DL) } \\
\text { (obm) }\end{array}$ \\
\hline$\overline{V W}$ & Stelt & 1328 & 7 & 28 & 11.9 & .276 \\
\hline TPlownlogd & 6tis? & 17.,48: & D & 23 & 96 & .104 .6 \\
\hline TPlownloat & 8te? & 1284 & $0.3 M$ & 21 & 918.5 & .41 \\
\hline Ved Bousing & stell & 133.77 & 0 & 2 & 0 & .100 .5 \\
\hline Vof & 6te? & 151.34 & t & 28 & 0 & .101 .12 \\
\hline TP Downoad & Sted?! & 157.60 & $8:$ & 28 & 60 & .1046 \\
\hline TPlownlogd & 6t:27! & 1033 & 0.142 & 2 & 93.2 & -10611 \\
\hline Plownoad & $6127\}$ & 16364 & & 22 & & .110 .4 \\
\hline
\end{tabular}

Fig 10(b): Dhaka Railway Traffic Map Simulation Properties

\begin{tabular}{|c|c|c|c|c|c|c|}
\hline Service & $\begin{array}{l}\text { Reference } \\
\text { Cell }\end{array}$ & $\begin{array}{l}\text { Total } \\
\text { Path } \\
\text { Loss } \\
\text { (DL) } \\
\text { (dB) }\end{array}$ & $\begin{array}{c}\text { Application } \\
\text { Channel } \\
\text { Throughput } \\
\text { (DL) } \\
\text { (kbps) }\end{array}$ & $\begin{array}{c}\text { Transmission } \\
\text { Power } \\
\text { (UL) } \\
\text { (dBm) }\end{array}$ & $\begin{array}{c}\text { Application } \\
\text { User } \\
\text { Throughput } \\
\text { (DL) } \\
\text { (kbps) }\end{array}$ & $\begin{array}{c}\text { Reference } \\
\text { Signal } \\
\mathrm{C} /(\mathrm{I}+\mathrm{II}) \\
\text { (DL) } \\
\text { (dB) }\end{array}$ \\
\hline$\overline{V O P}$ & Site14_1(0) & 159.65 & $3,741.86$ & 23 & 11.59 & -0.2 \\
\hline FTP Download & Sitte6_3 $3(0)$ & 154.8 & $34,549.34$ & 23 & 950.01 & 18.65 \\
\hline FTP Download & Site $33 \_2(0)$ & 164.64 & $7,645.71$ & 23 & 404.34 & 6.36 \\
\hline FTP Download & Site $42 \_1(0)$ & 143.48 & $5,702.75$ & 23 & 950 & 1.87 \\
\hline FTP Download & Site 33_1 (0) & 151.14 & 362.57 & 23 & 13.3 & -6.16 \\
\hline FTP Download & Site7_3 $(0)$ & 151.87 & 828.06 & 22.8 . & 350.44 & -3.93 \\
\hline Web Browsing & Sitteo_3 $3(0)$ & 135.79 & $7,313.29$ & 22.8 . & 121.6 & 3.81 \\
\hline FTP Download & Sitteo_1 $(0)$ & 146.48 & $7,313.29$ & 22.4 . & 950 & 6.33 \\
\hline FTP Download & Site7_3 $(0)$ & 153.24 & 947.28 & 23 & 400.9 & -4.03 \\
\hline Web Browsing & Site7_1 $(0)$ & 148.39 & $31,815.73$ & 23 & 121.6 & 17.33 \\
\hline FTP Download & Sites_2 $2(0)$ & 144.42 & $9,184.22$ & 21.7 & 950 & 9.04 \\
\hline Web Browsing & Sites_2 $2(0)$ & 140.88 & $34,549.34$ & 22.3 . & 121.6 & 19.01 \\
\hline FTP Download & Site3_1 (0) & 141.46 & $9,184.22$ & 21.5 & 950 & 9.28 \\
\hline FTP Download & Site7_1 (0) & 148.25 & $2,344,88$ & 23 & 741.65 & -1.47 \\
\hline FTP Download & Site7_1 (0) & 150.54 & $16,983.27$ & 23 & 950 & 13 \\
\hline FTP Download & Site7_2 (0) & 134.44 & $31,815.73$ & 21.4 . & 950 & 17.56 \\
\hline Web Browsing & Siteo_2 (0) & 151.86 & $24,271.68$ & 23 & 121.6 & 14.8 \\
\hline Web Browsing & Site6_1 (0) & 140.58 & $16,983.27$ & 23 & 121.6 & 12.85 \\
\hline FTP Download & Sitteo_2 (0) & 153.86 & $6,867.9$ & 23 & 892.14 & 3.35 \\
\hline FTP Download & Site6_1 $(0)$ & 149.05 & $34,549.34$ & 23 & 950.01 & 23.26 \\
\hline FTP Download & Site7_3 (0) & 121.49 & $8,220.91$ & 23 & 850.36 & 6.79 \\
\hline Web Browsing & Site7_3 (0) & 154.01 & $9,184.22$ & 22.7 . & 121.6 & 8.12 \\
\hline Web Browsing & Site7_3 $(0)$ & 158.92 & $9,184.22$ & 23 & 121.6 & 9.98 \\
\hline Web Browsing & Site7_3(0) & 142.11 & $34,549.34$ & 22.7 . & 121.6 & 19.05 \\
\hline Web Browsing & Sites_1 (0) & 143.71 & $9,184.22$ & 22.3 . & 121.6 & 8.39 \\
\hline
\end{tabular}

Fig 11(b): Dhaka Mainroad Traffic Map Simulation Properties

\subsection{Traffic Simulation}

LTE traffic simulation properties have been shown in Fig 8. Fig 9 to Fig 13 shows Dhaka airport, railway, mainroad, secondary road and street traffic maps after simulation (in (a)) along with their properties (in (b)). The properties chart shows service, reference cell, total pathloss, transmission power throughput and reference signal CINR. 


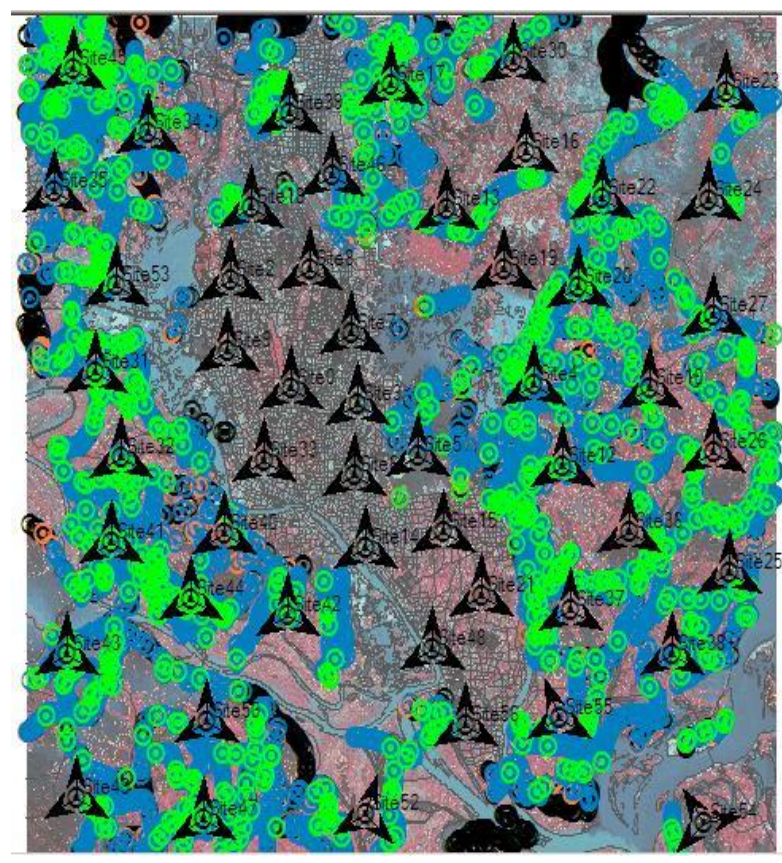

Fig 12(a): Dhaka Secondary Road Traffic Map after

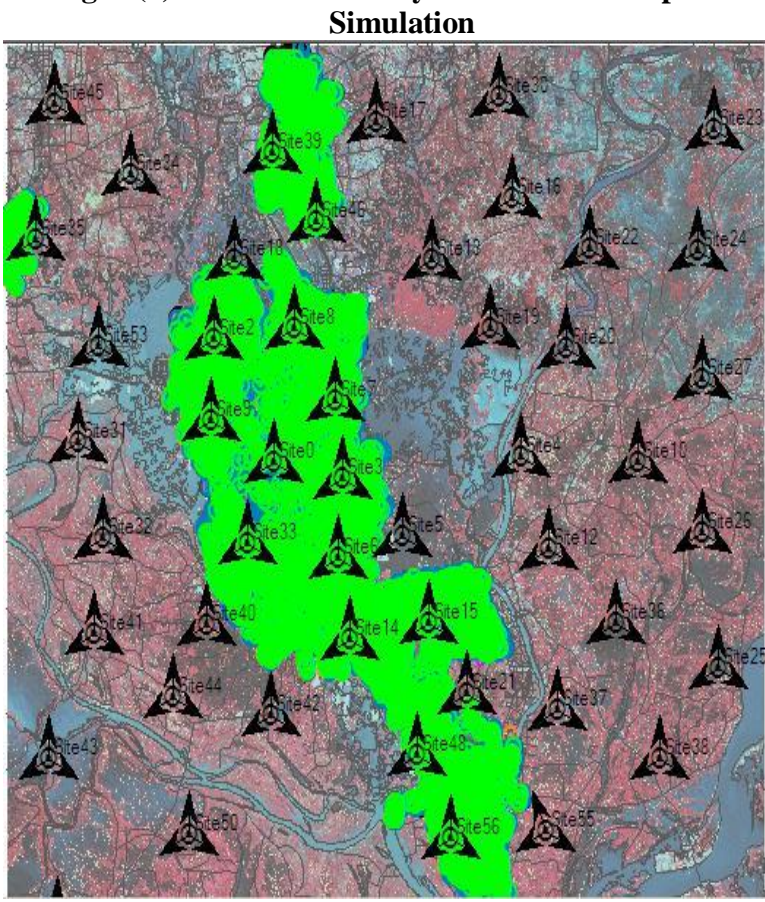

Fig 13(a): Dhaka Street Traffic Map after Simulation

\section{PERFORMANCE ANALYSIS OF THE PLANNED NETWORK}

Using point analysis tool of Atoll site 41_2 was chosen from the Dhaka map along with a receiver to analyze the cell edge throughput scenario and all other uplink and downlink parameters. The point analysis results appeared as the following shown in Fig 14 (a)-(e). Again, link budget scenario from the point analysis tool for another site 33_3 comes as the one shown in Table 1.This one almost matches with the prepared link budget and other obtained values using link and system simulators in [21].

\begin{tabular}{|c|c|c|c|c|c|}
\hline Service & $\begin{array}{l}\text { Reference } \\
\text { Cell }\end{array}$ & $\begin{array}{l}\text { Total } \\
\text { Path } \\
\text { Loss } \\
\text { (DL) } \\
\text { (dB) }\end{array}$ & $\begin{array}{c}\text { Application } \\
\text { Channel } \\
\text { Throughput } \\
\text { (DL) } \\
\text { (kbps) }\end{array}$ & $\begin{array}{l}\text { Application } \\
\text { User } \\
\text { Throughput } \\
\text { (DL) } \\
\text { (kbps) }\end{array}$ & $\begin{array}{c}\text { Received } \\
\text { Reference } \\
\text { Signal } \\
\text { Power } \\
\text { (DL) } \\
\text { (dBm) }\end{array}$ \\
\hline FTP Download & Site8_1 (0) & 157.72. & $1,358.92$ & 885.48 & -104.29 \\
\hline Web Browsing & Site5_1 (0) & 143.94 & $7,313.29$ & 121.6 & -90.51 \\
\hline FTP Download & Sites_1 (0) & 146.19: & $9,457.13$ & 183.93 & -92.76 \\
\hline FTP Download & Sites_1 (0) & 132.02 & $11,905.4$ & 231.55 & -78.59 \\
\hline Web Browsing & Sites_1 (0) & 144.75 & $3,741.86$ & 121.6 & -91.32 \\
\hline FTP Download & Sites_1 (0) & 145.41 & $28,134.82$ & 547.19 & -91.98 \\
\hline FTP Download & Sites_1 (0) & 158.22 & $2,344.88$ & 45.61 & -104.79 \\
\hline FTP Download & Site14_3(0) & 146.48 & $7,313.29$ & 258.49 & -93.05 \\
\hline FTP Download & Site $40 \_3(0)$ & 153.4 & $9,184.22$ & 690.77 & -99.97 \\
\hline Web Browsing & Site $40 \_2(0)$ & 151.49 & $5,454.8$ & 121.6 & -98.06 \\
\hline Web Browsing & Site $40 \_2(0)$ & 147.17 & $9,184.22$ & 121.6 & -93.74 \\
\hline FTP Download & Site $40 \_2(0)$ & 150.26 & $34,549,34$ & 950.01 & -96.82 \\
\hline FTP Download & Site $44 \_1(0)$ & 147.2 & $4,453.67$ & 94.96 & -93.98 \\
\hline FTP Download & Site $40 \_2(0)$ & 156.74 & $20,664.17$ & 950 & -103.31 \\
\hline FTP Download & Site $44 \_1(0)$ & 155.27 . & $9,601.68$ & 204.74 & -102.06 \\
\hline VolP & Site $32 \_2(0)$ & 151.88 & 766.73 & 8.97 & -101.67 \\
\hline FTP Download & Site $40 \_1(0)$ & 134.1 & $34,549.34$ & 950.01 & -80.67 \\
\hline FTP Download & Site $40 \_1(0)$ & 138.61 & $6,751.42$ & 333.85 & -85.18 \\
\hline FTP Download & Site $40 \_1(0)$ & 151.7 & $16,373.33$ & 809.65 & -98.27 \\
\hline Web Browsing & Site $40 \_3(0)$ & 140.05 & $9,184.22$ & 121.6 & -86.62 \\
\hline FTP Download & Site $40 \_3(0)$ & 140.9 & $28,134,82$ & 950 & -87.47 \\
\hline Web Browsing & Site $40 \_3(0)$ & 143.38. & $26,349.6$ & 113.89 & -89.95 \\
\hline FTP Download & Site $44 \_1(0)$ & 151.47 . & 990.34 & 21.12 & -98.25 \\
\hline Web Browsing & Site $40 \_2(0)$ & 152.79: & $2,657.06$ & 86.35 & -99.36 \\
\hline FTP Download & Site $42 \_1(0)$ & 152.98. & $7,645.71$ & 108.06 & -99.76 \\
\hline
\end{tabular}

Fig 12(b): Dhaka Secondary Road Traffic Map Simulation Properties

\begin{tabular}{|c|c|c|c|c|c|c|}
\hline Service & $\begin{array}{l}\text { Reference } \\
\text { Cell }\end{array}$ & $\begin{array}{l}\text { Total } \\
\text { Path } \\
\text { Loss } \\
\text { (DL) } \\
\text { (dB) }\end{array}$ & $\begin{array}{c}\text { Application } \\
\text { Channel } \\
\text { Throughput } \\
\text { (DL) } \\
\text { (kbps) }\end{array}$ & $\begin{array}{c}\text { Transmission } \\
\text { Power } \\
\text { (UL) } \\
\text { (dBm) }\end{array}$ & $\begin{array}{c}\text { Application } \\
\text { User } \\
\text { Throughput } \\
\text { (DL) } \\
\text { (kbps) }\end{array}$ & $\begin{array}{l}\text { Reference } \\
\text { Signal } \\
\mathrm{C} /(\mathrm{I}+\mathrm{N}) \\
\text { (DL) } \\
\text { (dB) }\end{array}$ \\
\hline VolP & Site33_1 (0) & 131.05 & $29,233.32$ & 23 & 11.52 & 16.09 \\
\hline VolP & Siteo_3 $(0)$ & 148.31 & $2,344.88$ & 23 & 11.59 & -1.89 \\
\hline VolP & Siteo_3 $(0)$ & 149.77 & $9,184.22$ & 21.8 & 11.59: & 9.05 \\
\hline VolP & Site33_1 (0) & 155.51 & $9,601.68$ & 23 & 11.59 & 7.3 \\
\hline VolP & Site33_1 (0) & 149.75 & 979.8. & 22.7 . & 11.47. & -5.22 \\
\hline VolP & Siteo_3 $3(0)$ & 148.79 & $5,454.8$ & 22.9 & 11.59: & 2.37 \\
\hline Volp & Site9_2(0) & 144.87 & $5,454.8$ & 23 & 11.59 & 1.97 \\
\hline VolP & Siteo_3 $(0)$ & 153.11 & $5,707.35$ & 22.2 & 9.05 & 3.07 \\
\hline FTP Download & Site9_2(0) & 143.45 & $2,344.88$ & 23. & 0 & -1.53 \\
\hline VolP & Siteo_2 $(0)$ & 155.62 & $3,124.55$ & 23. & 9.68 & -0.87 \\
\hline VolP & Siteo_2 (0) & 152 & $7,313.29$ & 22.3 & 11.59 & 4.78 \\
\hline VolP & Siteo_2 (0) & 160.5 & 34,549.34: & 23 & 11.59: & 18.85 \\
\hline VolP & Siteo_2 $(0)$ & 159.29 & $9,184.22$ & 23 & 11.59 & 7.48 \\
\hline VolP & Site33_1 (0) & 164.29 & 649.12 & 23 & 7.6 & -5.78 \\
\hline VolP & Siteo_2 (0) & 147.11 & $2,344.88$ & 23 & 11.59 & -1.61 \\
\hline Video Conferencing & Site3_3(0) & 155.3 & $3,025.68$ & 22.5 & 49.16: & -0.89 \\
\hline VolP & Site3_3 (0) & 155.12 & 11,644.85: & 23 & 11.34 & 10.41 \\
\hline VolP & Site3_3(0) & 150.38 & $24,271.68$ & 23 & 11.59 & 14.46 \\
\hline VolP & Ste3_3(0) & 155.23 & $8,020.89$ & 23. & 10.12 & 6.75 \\
\hline VolP & Site6_1 $(0)$ & 147.99 & $7,313.29$ & 23 & 11.59 & 3.98 \\
\hline VolP & Siteo_2 (0) & 139.69 & $24,271.68$ : & 22.7 & 11.59 & 15.13 \\
\hline VolP & Siteo_2 (0) & 150.29 & $3,741.86$ & 23 & 11.59 & 0.13 \\
\hline VolP & Siteo_2(0) & 142.31 & $9,184.22$ & 21.7. & 11.59: & 9.08 \\
\hline VolP & Siteo_2 (0) & 136.15 & $34,549.34$ & 23 & 11.59 & 20.8 \\
\hline VolP & Siteo_1 (0) & 139.84 & $8,659.73$ & 23 & 10.93 & 6.97 \\
\hline
\end{tabular}

Fig 13(b): Dhaka Street Traffic Map Simulation Properties

Fig.14 (b): shows the geographic profile of the site 41_2, (c) shows the reception level including the adjacent sites, (d) gives the signal analysis involving SCH \& PBCH, Downlink and Uplink parameters of the adjacent sites while (e) provides the result taking the comparatively better transmitters into account. 

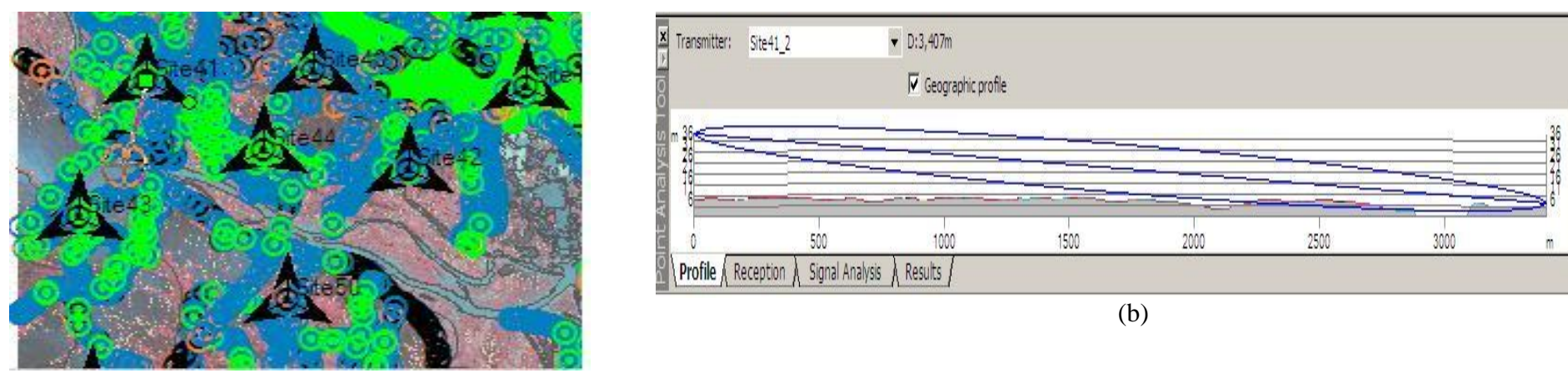

(b)

(a)

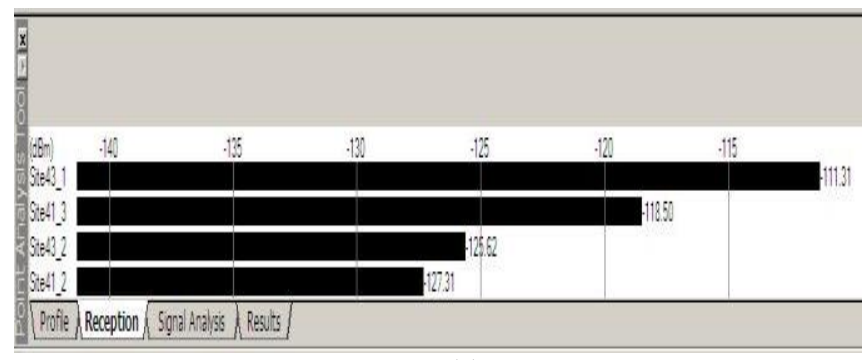

(c)

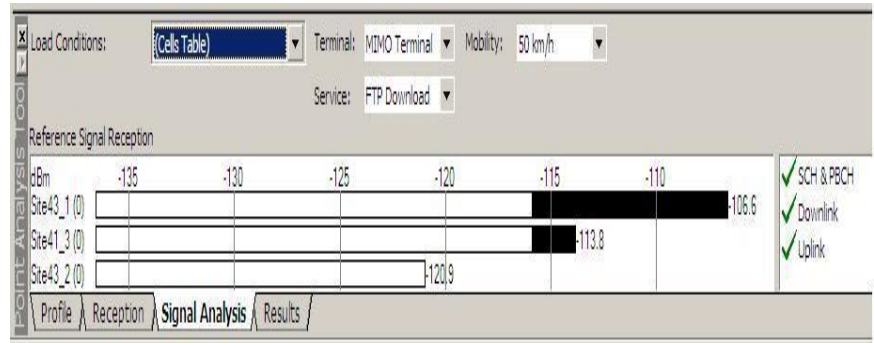

(d)

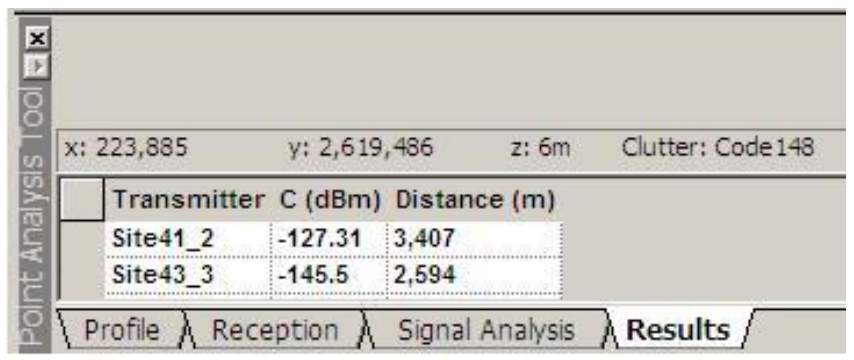

(e)

Fig 14: Chosen site and receiver for point analysis and performance result-(a), (b), (c), (d), (e)

\section{Table 1: Link budget Obtained from point analysis tool}

$\begin{array}{ll}\text { Transmitter: } & \text { Site33_3 } \\ \text { Receiver: } & (230,172 ; 2,626,698) \\ \text { Distance: } & 2,571 \mathrm{~m} \\ \text { Power: } & 43 \mathrm{dBm} \\ \text { EIRP: } & 53.22 \mathrm{dBm} \\ \text { Reception gain: } & 0 \mathrm{~dB} \\ \text { Path Loss: } & 160.01 \mathrm{~dB} \\ \text { Shadowing margin: } & 4.72 \mathrm{~dB} \\ & \\ \text { Indoor Loss: } & \text { O dB } \\ & \\ \text { Signal Level: } & -111.51 \mathrm{dBm}\end{array}$

Analyzing the coverage prediction results with the placed eNodeB with respect to [21] it is quite evident that the planned network provides a satisfactory coverage. Again, evaluation of traffic map after simulation makes it clear that subscribers mostly remain connected at both UL \& DL which also indicates a very positive sign for the planned network. Performance analysis with point analysis tool strengthens the base behind the planned network as an effective one. 


\section{CONCLUSION AND FUTURE WORK}

The ultimate objectives of the present study of LTE radio network planning guidelines are to introduce the relevant LTE features, to define the basic models for radio propagation planning, to estimate coverage and network element count. The prepared guideline may assist in the development of various tools used in Radio Network Planning (RNP). Obtained result of coverage and capacity analysis has been used in nominal and detailed radio planning stage with Atoll taking Dhaka digital map as input. In detail Atoll simulations have been run on Dhaka digital map containing both coverage predictions and traffic simulations. Again, performance evaluation has been done using point analysis tool. For initial network deployment, at the very beginning only a small number of subscribers are considered for coverage and capacity calculation. So, there remains the challenge for future capacity enhancement. But still it can be considered as a standard radio planning platform for the densely populated South-Asian city Dhaka.

\section{ACKNOWLEDGMENTS}

Our heartiest thanks go to Mohammad Tawhid Kawser, Assistant Prof., EEE Dept., IUT and Mohammad Nur-AAlam, Solution Manager, Nokia Siemens Network (NSN) for providing numerous help and support in this work.

\section{REFERENCES}

[1] LTE - The UMTS Long Term Evolution From Theory to Practice by From Theory to Practice by Stefania Sesia, Issam Toufik and Matthew Baker.

[2] LTE for UMTS OFDMA and SC-FDMA Based Radio Access by Harry Holma and Antti Toskala.

[3] Long Term Evolution (LTE): A Technical Overview Technical White paper by Motorola.

[4] White paper: "Long Term Evolution Protocol Overview" by freescale semiconductor.

[5] White paper: "LTE-An Introduction" by Ericsson.

[6] Abdul Basit, Syed-"Dimensioning of LTE Network Description of Models and Tool, Coverage and Capacity Estimation of 3GPP Long Term Evolution radio interface " Masters Thesis submitted in Helsinki University of Technology

[7] Basanta Shrestha- "LTE Radio Network Performance Analysis"- Master of Science Thesis of Tampere University of Technology.

[8] Hosein, P."Resource Allocation for the LTE Physical Downlink Control Channel"-GLOBECOM Workshops, 2009, IEEE.

[9] C. Mehlführer, M. Wrulich, J. Colom Ikuno, D Bosanska and M. Rup, "Simulating the Long Term Evolution Physical Layer," in Proc. EUSIPCO 2009 p. $1471-1478$.
[10] 3GPP Technical Specification 36.101, 'User Equipment (UE) Radio Transmission and Reception (Release 8)', www.3gpp.org.

[11] 3GPP Technical Specification 36.104, 'Base Station (BS) Radio Transmission and Reception (Release 8)', www.3gpp.org.

[12] 3GPP Technical Specification 36.211, 'Physical Channels and Modulation (Release 8)', www.3gpp.org.

[13] 3GPP Technical Specification 36.213, 'Physical layer procedures (Release 8)', www.3gpp.org.

[14] 3GPP Technical Specification 36.214, 'Evolved Universal Terrestrial Radio Access (E-UTRA); Physical Layer - Measurements (Release 8)', www.3gpp.org.

[15] 3GPP TS 36.322 V8.4.0(2008-12) "Evolved Universal Terrestrial Radio Access (E-UTRA); Radio Link Control (RLC) protocol specification"

[16] 3GPP TS 36.321 V8.5.0 (2009-03)-"Evolved Universal Terrestrial Radio Access (E-UTRA); Medium Access Control (MAC) protocol specification".

[17] LTE Link Level Simulator http://www.nt.tuwien.ac.at/about-us/staff/josep-colomikuno/lte-link-level-simulator/

[18] LTE System Level Simulator http://www.nt.tuwien.ac.at/about-us/staff/josep-colomikuno/lte-system-level-simulator/

[19] Mohammad Kawser, Nafiz Imtiaz Bin Hamid, Md. Nayeemul Hasan, Md. Shah Alam and Md. Musfiqur Rahman - "Downlink SNR to CQI Mapping for Different Multiple Antenna Techniques in LTE"International Conference on Future Information Technology (ICFIT), Changsha, China, December, 2010.

[20] Nafiz Imtiaz Bin Hamid, Md. R. H. Khandokar, Taskin Jamal, Md.A. Shoeb and Md. Zakir Hossain - "In Quest of the Better Mobile Broadband Solution for South Asia Taking WiMAX and LTE into Consideration" - Journal of Telecommunications (ISSN: 2042-8839), Vol.2 Issue 1, April 2010. pp.86-94.

[21] Nafiz Imtiaz Bin Hamid, Mohammad T. Kawser, Md. Ashraful Hoque- "Coverage and Capacity Analysis of LTE Radio Network Planning considering Dhaka City"International Journal of Computer Application (IJCA)Vol.46, No.15, May 2012. pp.49-56. 